\section{Why should apparently healthy children engage in physical activity?}

Lars Bo Andersen

WHO has recommended 60 minutes of moderate physical activity per day in children. The recommendations are mainly based on evidence of the benefits on metabolic health even if other health parameters are just as important. Hallal et al. published worldwide prevalence of physical inactivity in children in 2012 and stated that more than $80 \%$ of all children did not fulfil guidelines ${ }^{1}$. This estimate is based on self-reported physical activity and when objective measures are used the number of inactive children depends highly on which cutpoints are used to define what moderate intensity physical activity corresponds to in accelerometer output. Most children do not show any visual signs of an unhealthy condition, which could be related to physical inactivity, and do not perceive they are ill. For not more than one or two decades ago researchers struggled to define health in children and if the health status cannot be assessed accurately, it is very difficult to analyze how much physical activity children need in order to stay healthy. Many studies were published where the associations between physical activity and different metabolic biomarkers were assessed. However, associations were rather weak and it was difficult to define a healthy level for each biomarker above which risk for future disease was increased. Some attempts were made to translate definitions of metabolic syndrome in adults into cutpoints in the same risk factors which could be used in children. This was not very successful, because these definitions only selected less than $1 \%$ of most pediatric populations with metabolic syndrome, and this number compares poorly with $80 \%$ being inactive.

In the mid 1990s we started to look at metabolic health on a continuous scale and constructed composite scores from the known cardiovascular risk factors. This tool could not be used to define which children were healthy, but it improved associations between physical activity and health status. The concept was later optimized by using $\mathrm{z}$-scores for the different risk factors ${ }^{2}$. At the same time we developed a different approach to define which children had an unhealthy metabolic profile based on the same observation as Peter Reaven had when he defined metabolic syndrome in the mid 1980s. He observed that all the risk factors for some reason were elevated in the same person, and the elevation was most likely caused by insulin resistance. Metabolic risk factor levels are lower in children than in adults, but clustering of risk factors in the same child was apparent, and this elevation in many risk factors compared to levels in peers of same age and sex could probably accelerate atherosclerosis and therefore be defined as an unhealthy state. Number of children with clustering of risk factors in a population can be calculated, because the number of children with a specific number of risk factors would follow a binominal distribution if they were independent of each other. Using this approach to define ill health, we found that clustering was apparent in around $10 \%$ of children in populations from different parts of the world ${ }^{3}$.

Based on the overwhelming literature of increasing obesity trends worldwide, one might expect that obesity was responsible for clustering of risk
Rev Bras Ativ Fis Saúde p. 445-446 $\mathrm{DOI}$

http://dx.doi.org/10.12820/rbafs.v.20n4p445

Sogn and Fjordane University College, Sogndal, Norway 
factors in these children. However, this is only partly true. We have found that low fitness is an even stronger predictor of clustered CVD risk than obesity. In the official definitions of metabolic syndrome published by International Diabetes Federation (IDF) and WHO, fitness is not even included in the definition. However, we find normal weight children with clustering of CVD risk factors in our studies. This is not surprising because muscle tissue is the largest organ in the body and if a child is insulin resistant, it will affect glucose uptake in muscles. In fact, one-leg training studies have shown that insulin sensitivity can be doubles in a few months in the trained leg compared with the untrained leg. Much evidence points against physical inactivity in a key role in the development of metabolic disorders. Physical activity is not very effective in weight loss strategies, but this does not mean that changes are not happening in other parameters. A recent randomized controlled trial the intervention group decreased physical activity in young healthy subjects to less than 1500 steps per day compared with $>10,000$ in the control group, and they could in just two weeks measure increased abdominal fat assessed by MRI scan in the sedentary group.

Promotion of physical activity in children can include the whole population, because 'treating' healthy individuals has no side effects. However, it may be necessary specifically to target children with clustered CVD risk if health should be improved in these children. In order to do that, it is necessary to have simple tools to diagnose children with metabolic disorder. This is much easier than we should expect. We have observed that when we construct a composite risk factor score, this score is not gradually increasing when subjects are ranked according to their score. In a ranked population the score increases gradually to a certain level and then it takes off, which means that we can easily select children with an extreme score. The commonly used composite score consist of the risk factors from the metabolic syndrome, and this score will not be feasible as a screening tool because it includes blood sampling. We therefore constructed a score based only on waist per height and the inverse of aerobic fitness. Waist and height are easily measured and fitness can be assessed in a whole school class during one physical education lesson using an indirect field test. We found that this simple non-invasive measure had specificity and sensitivity against the IDF definition of metabolic syndrome $>0.85^{3}$.

Future promotion of physical activity in children can be targeted against the whole population and the school arena will probably be the best way to get sedentary children included. However, it is also possible to target children at risk, and this should not just include obese children. Sedentary children may need it just as much even if it is not visible on the outside of the body.

\section{REFERENCES}

1. Hallal PC, Andersen LB, Bull F, Guthold R, Haskell WL, the Lancet Physical Activity Series Working Group. Global physical activity levels: past, present, and future. Lancet in press. 2012.

2. Andersen LB, Harro M, Sardinha LB, Froberg K, Ekelund U, Brage S et al. Physical activity and clustered cardiovascular risk in children: a cross-sectional study (The European Youth Heart Study). Lancet. 2006; 368(9532):299-304.

3. Andersen LB, Lauersen JB, Brond JC, Anderssen SA, Sardinha LB, Steene-Johannessen $\mathrm{J}$ et al. A new approach to define and diagnose cardiometabolic disorder in children. J Diabetes Res. 2015; 2015:539835. 